\title{
Hiding in the dark: uncovering cancer drivers through image-guided genomics
}

\author{
Kosuke Yoshihara ${ }^{1 *}$ and Roel GW Verhaak ${ }^{2,3^{*}}$ \\ See related research, http://genomebiology.com/2014/15/12/526
}

\begin{abstract}
Genome analysis which takes into account tumor purity leads to discovery of PTEN as a tumor suppressor gene in high-grade serous ovarian cancer.
\end{abstract}

High-throughput approaches for molecular characterization of biospecimens enable us to comprehensively dissect cancer cells. Cohort studies of clinical tumor samples have provided valuable new insights, such as the discovery of genes that drive cancer [1]. However, surgical resection of tumor tissue often results in the inclusion of tumoradjacent normal cells and a tumor microenvironment. As a consequence, the tissue samples under investigation in genomic and transcriptomic studies reflect heterogeneity in the number of tumor cells actually analyzed [2]. Tumor purity may be an important determinant of our ability to further uncover driver genes. In this issue of Genome Biology, Brenton and colleagues aimed to account for tumor purity by estimating stromal content in an unbiased fashion using in silico hematoxylin and eosin (H\&E) slide image analysis [3].

\section{Defogging the ovarian carcinoma genome}

The tumor microenvironment consists of immune cells, endothelial cells and fibroblasts, and is commonly referred to as tumor stroma. The stromal component contributes to the pool of DNA and RNA molecules that are used for genomic/transcriptomic analysis. While mutation analysis is generally less affected as sequencing depth can account for the relative proportion of tumor DNA, the diluting effect of non-tumor cells may be more profound on DNA

\footnotetext{
* Correspondence: yoshikou@med.niigata-u.ac.jp; rverhaak@mdanderson.org 1 Department of Obstetrics and Gynecology, Niigata University Graduate School of Medical and Dental Sciences, Niigata 951-8510, Japan

${ }^{2}$ Department of Bioinformatics and Computational Biology, The University of Texas MD Anderson Cancer Center, Houston, TX 77030, USA

${ }^{3}$ Department of Genome Medicine, The University of Texas MD Anderson Cancer Center, Houston, TX 77030, USA.
}

copy number analysis. Genes that are homozygously deleted in the tumor cells may appear as single copy loss due to the admixture of stromal content. The same stromal component may express the gene, thus creating the impression of a hemizygously lost gene that is actively being transcribed. In order to avoid the analysis of tissue samples with very low numbers of tumor cells, tumor purity is routinely assessed. The classical and clinically widely used method is the manual evaluation of tissue sections embedded on glass slides treated with an H\&E dye that visualizes individual cells and cell structures. By coloring otherwise transparent tissue sections, microscopy viewing of $\mathrm{H} \& \mathrm{E}$-stained slides allows experienced pathologists and researchers to distinguish different cell types, including cancer cells, immune cells and fibroblasts. Unfortunately, this approach is affected by inter- and intra-observer biases [4]. The inclusion of tumor stroma can be avoided by using methods such as laser-capture microdissection and highthroughput cell sorting to isolate tumor cells, but these approaches are time intensive and resource consuming and for these reasons non-practical for clinical use and have only limited applicability in the research setting.

Brenton and colleagues reasoned that an automated approach may lead to higher reproducibility and accuracy of tumor purity prediction compared with manual assessment. Immunohistochemistry stains of high-grade serous ovarian cancer were matched with various molecular profiles that are available through The Cancer Genome Atlas (TCGA) [1] and comparison of the image-based stromal tissue estimates to those generated by a published and validated mRNA signature based method [2] showed a significant correlation, which suggested the accuracy of their approach. In parallel, they performed semi-automated analysis of ovarian carcinoma tissue microarray images to associate tumor-specific phosphatase and tensin homo$\log$ (PTEN) protein expression levels with clinical outcome in two independent large cohorts of ovarian cancer (Study of Epidemiology and Risk Factors in Cancer Heredity (SEARCH); Nottingham Ovarian Cancer Study (NOT)) 
that were part of the Ovarian Tumor Tissue Analysis consortium study [5]. The tissue microarrays provided sufficient detail to allow scoring of PTEN expression in tumor cells relative to that in stromal cells. As a result, low absolute levels of tumor-specific PTEN expression were observed in $77 \%$ of the SEARCH cohort and 52\% of the NOT cohort. Moreover, the authors showed that reduced PTEN expression was significantly and independently associated with clinical outcome as well as PTEN copy number status in ovarian cancer. In summary, these findings suggested that PTEN is a putative tumor suppressor gene in ovarian carcinoma.

The results by Brenton and colleagues highlight the value of integrating $H \& E$ slide analysis, tumor purity and genomic/transcriptomic analysis. One potential caveat of this approach may be that the tissue sections used for image analysis and genomic/transcriptomic analysis may differ, resulting in discordant results. Ideally, H\&E-stained slides should be obtained from tumor areas in close proximity to tumor tissue used for genomic/transcriptomic analysis.

\section{Clinical significance of PI3K/AKT signaling}

Deactivation of PTEN through somatic mutations and deletions is a common driver event of many cancers and results in hyperactivation of the PI3K/AKT pathway. The PI3K/AKT pathway regulates cell proliferation, survival, and energy metabolism and key members such as PI3KCA, $A K T 1, A K T 2, A K T 3, m T O R C 1$ and $m T O R C 2$ provide attractive therapeutic targets. DNA copy number analysis by TCGA has suggested that focal amplifications of PIK3CA, $A K T 1, A K T 2$ and $A K T 3$ are frequent events in high-grade serous ovarian carcinoma and provided earlier indications that this pathway is commonly altered in this disease. However, while functional studies confirmed the activation of the PI3K/AKT axis through genetic alterations in ovarian carcinoma, this does not ubiquitously confer sensitivity to inhibitory signals, suggesting functional redundancy of the pathway [6]. Acquired inactivation of PTEN can provide resistance to PI3K $\alpha$ inhibitors [7] and may present an example of how disease critical pathways can be double wired.

The TCGA analysis revealed that high-grade serous ovarian cancer is characterized by TP53 mutation (96\%), homologous recombination deficiency $(51 \%)$ and somatic or germline mutation of BRCA1 and BRCA2 (20\%) [1]. The frequent deactivation of homologous recombination pathways led to excitement over the possible application of poly (ADP-ribose) polymerase (PARP) inhibitors for treatment of ovarian carcinomas. PARP inhibitors block DNA single-strand break repair, resulting in systematic lethality with homologous recombination deficiency and are being tested in ovarian cancer clinical trials using $B R C A 1 / 2$ status as a biomarker (see ClinicalTrials.gov:
NCT00753545, NCT01874353 and NCT01844986) [8]. PTEN loss also causes defects in repair of DNA doublestrand breaks by homologous recombination and may provide an alternative mechanism of sensitivity to PARP inhibitors [9]. Interestingly, combination of PARP and PI3K inhibitors showed a synergistic antitumor effect in PTEN-deficient prostate tumors [10]. A phase I study of the PI3K inhibitor BKM120 and PARP inhibitor olaparib in patients with recurrent triple-negative breast cancer and recurrent high-grade serous ovarian cancer is underway (NCT01623349) and will provide important early clinical data on whether this combination therapy may provide a therapeutic advance. The standard of care for patients with high-grade ovarian cancer has not changed in several decades except for the relatively recent addition of angiogenesis inhibitors such as bevacizumab. The establishment of novel treatment modalities aimed at molecular targets may lead to improvement of prognosis for patients in dire need of better outcomes. Tumorspecific PTEN protein expression may provide one aspect of a multibiomarker panel that may guide the selection of optimal therapy to inhibit the PI3K/AKT pathway.

\section{Concluding remarks}

A combination of genomic and image analyses has shed light on the abrogation of PTEN which was hiding behind a shade of tumor stroma and showed that loss of PTEN function can be a driver event and prognostic factor in high-grade serous ovarian cancer. These findings encourage us to re-evaluate the detection of tumor suppressor genes after taking into consideration tumor purity.

\section{Abbreviations}

AKT1: v-akt murine thymoma viral oncogene homolog 1; AKT2: v-akt murine thymoma viral oncogene homolog 2; AKT3: v-akt murine thymoma viral oncogene homolog 3; BRCA1: Breast cancer 1, early onset; BRCA2: Breast cancer 2, early onset; $m T O R C 1$ : Mechanistic target of rapamycin, complex 1; mTORC2: Mechanistic target of rapamycin, complex 2; NOT: Nottingham Ovarian Cancer Study; PARP: Poly (ADP-ribose) polymerase;

PI3K: Phosphatidylinositol-4,5-bisphosphate 3-kinase; PTEN: Phosphatase and tensin homolog; SEARCH: Study of Epidemiology and Risk Factors in Cancer Heredity; TCGA: The Cancer Genome Atlas; TP53: Tumor protein p53.

\section{Competing interests}

The authors declare that they have no competing interests.

\section{Published online: 20 December 2014}

\section{References}

1. The Cancer Genome Atlas Research Network: Integrated genomic analyses of ovarian carcinoma. Nature 2011, 474:609-615.

2. Yoshihara K, Shahmoradgoli M, Martinez E, Vegesna R, Kim H, Torres-Garcia W, Trevino V, Shen H, Laird PW, Levine DA, Carter SL, Getz G, Stemke-Hale K, Mills GB, Verhaak RG: Inferring tumour purity and stromal and immune cell admixture from expression data. Nat Commun 2013, 4:2612.

3. Martins FC, de Santiago I, Tring A, Xian J, Guo A, Sayal K, Jimenez-Linan M, Deen S, Driver K, Mack M, Aslop J, Pharoah PD, Markowetz F, Brenton JD: Combined image and genomic analysis of high-grade serous ovarian cancer reveals PTEN loss as a common driver event and prognostic classifier. Genome Biol 2014, 15:526. 
4. Smits AJ, Kummer JA, de Bruin PC, Bol M, van den Tweel JG, Seldenrijk KA, Willems SM, Offerhaus GJ, de Weger RA, van Diest PJ, Vink A: The estimation of tumor cell percentage for molecular testing by pathologists is not accurate. Mod Pathol 2014, 27:168-174.

5. Sieh W, Kobel M, Longacre TA, Bowtell DD, deFazio A, Goodman MT, Hogdall E, Deen S, Wentzensen N, Moysich KB, Brenton JD, Clarke BA, Menon U, Gilks CB, Kim A, Madore J, Fereday S, George J, Galletta L, Lurie G, Wilkens LR, Carney ME, Thompson PJ, Matsuno RK, Kjær SK, Jensen A, Høgdall C, Kalli KR, Fridley BL, Keeney GL, et al: Hormone-receptor expression and ovarian cancer survival: an Ovarian Tumor Tissue Analysis consortium study. Lancet Oncol 2013, 14:853-862.

6. Hanrahan AJ, Schultz N, Westfal ML, Sakr RA, Giri DD, Scarperi S, Janakiraman M, Olvera N, Stevens EV, She QB, Aghajanian C, King TA, Stanchina E, Spriggs DR, Heguy A, Taylor BS, Sander C, Rosen N, Levine DA, Solit DB: Genomic complexity and AKT dependence in serous ovarian cancer. Cancer Discov 2012, 2:56-67.

7. Juric $\mathrm{D}$, Castel $\mathrm{P}$, Griffith $\mathrm{M}$, Griffith $\mathrm{OL}$, Won $\mathrm{HH}$, Ellis $\mathrm{H}$, Ebbesen $\mathrm{SH}$, Ainscough BJ, Ramu A, lyer G, Shah RH, Huynh T, Mino-Kenudson M, Sgroi D, Isakoff S, Thabet A, Elamine L, Solit DB, Lowe SW, Quadt C, Peters M, Derti A, Schegel R, Huang A, Mardis ER, Berger MF, Baselga J, Scaltriti M: Convergent loss of PTEN leads to clinical resistance to a PI(3)Kalpha inhibitor. Nature 2014, doi: 10.1038/nature13948.

8. Ledermann J, Harter P, Gourley C, Friedlander M, Vergote I, Rustin G, Scott CL, Meier W, Shapira-Frommer R, Safra T, Matei D, Fielding A, Spencer S, Dougherty B, Orr M, Hodgson D, Barrett JC, Matulonis U: Olaparib maintenance therapy in patients with platinum-sensitive relapsed serous ovarian cancer: a preplanned retrospective analysis of outcomes by BRCA status in a randomised phase 2 trial. Lancet Oncol 2014, 15:852-861.

9. Dedes K, Wetterskog D, Mendes-Pereira AM, Natrajan R, Lambros MB, Geyer FC, Vatcheva R, Savage K, Mackay A, Lord CJ, Ashworth A, Reis-Filho JS: PTEN deficiency in endometrioid endometrial adenocarcinomas predicts sensitivity to PARP inhibitors. Sci Trans/ Med 2010, 2:53ra75.

10. Gonzalez-Billalabeitia E, Seitzer N, Song SJ, Song MS, Patnaik A, Liu XS, Epping MT, Papa A, Hobbs RM, Chen M, Lunardi A, Ng C, Webster KA, Signoretti S, Loda M, Asara JM, Nardella C, Clohessy JG, Cantley LC, Pandolfi PP: Vulnerabilities of PTEN-TP53-deficient prostate cancers to compound PARP-PI3K inhibition. Cancer Discov 2014, 4:896-904.

doi:10.1186/s13059-014-0563-3

Cite this article as: Yoshihara and Verhaak: Hiding in the dark: uncovering cancer drivers through image-guided genomics. Genome Biology 2014 15:563. 